\title{
Short Communication \\ Cigarette smoking and risk of acoustic neuromas and pituitary tumours in the Million Women Study
}

\author{
VS Benson*,', J Green', K Pirie' and V Beral', for the Million Women Study Collaborators² \\ 'Cancer Epidemiology Unit, Nuffield Department of Clinical Medicine, University of Oxford, Richard Doll Building, Roosevelt Drive, Oxford OX3 7LF, UK
}

BACKGROUND: The relationship between cigarette smoking and incidence of acoustic neuromas and pituitary tumours is uncertain. METHODS: We examined the relation between smoking and risk of acoustic neuromas and pituitary tumours in a prospective study of 1.2 million middle-aged women in the United Kingdom.

RESULTS: Over 10.2 million person years of follow-up, 177 women were diagnosed with acoustic neuromas and 174 with pituitary tumours. Current smokers at recruitment were at significantly reduced risk of incident acoustic neuroma compared with never smokers (adjusted relative risk $(R R)=0.4 I$, 95\% confidence interval $(C l)=0.24-0.70, P=0.00 I$ ). Past smokers did not have significantly different risk of acoustic neuroma than never smokers $(R R=0.87,95 \% \mathrm{Cl}=0.62-1.22, P=0.4)$. Smoking was not associated with incidence of pituitary tumours (RR in current vs never smokers $=0.91,95 \% \mathrm{Cl}=0.60-1.40, P=0.7$ ).

CONCLUSION: Women who smoke are at a significantly reduced risk of acoustic neuromas, but not of pituitary tumours, compared with never smokers. Acoustic neuromas are much rarer than the cancers that are increased among smokers.

British Journal of Cancer (2010) I 02, 1654- 1656. doi:I0.1038/sj.bjc.6605695 www.bjcancer.com

Published online II May 2010

(c) 2010 Cancer Research UK

Keywords: acoustic neuroma; pituitary tumour; smoking; women

Acoustic neuromas and pituitary tumours are rare, each accounting for approximately $10 \%$ of all intracranial tumours. Acoustic neuromas are slow-growing benign tumours arising from Schwann cells of the eighth cranial nerve (Propp et al, 2006). Pituitary tumours are typically benign adenomas and may be hormone secreting or non-functioning (Asa and Ezzat, 2009). The aetiology of both tumour types is unclear (Evans et al, 2005; McGregor, 2009), with no firmly established common environmental risk factors. However, recent results from a multicentre case-control study showed a significantly lower risk for acoustic neuroma in current smokers than in never smokers (Schoemaker et al, 2007); no association was found between smoking and incidence of pituitary tumour (Schoemaker and Swerdlow, 2009).

We examined the relation between smoking and incident acoustic neuromas and pituitary tumours in a large prospective study of middle-aged women in the United Kingdom.

\section{MATERIALS AND METHODS}

During 1996-2001, 1.3 million middle-aged women (mean age 56 years) were recruited into the Million Women Study cohort, completing a recruitment questionnaire about socio-demographic factors, reproductive and medical history and other personal characteristics, including details on smoking status. Full details of

*Correspondence: Dr VS Benson; E-mail: vicky.benson@ceu.ox.ac.uk

${ }^{2}$ See Appendix

Received 10 December 2009; revised 14 April 2010; accepted 16 April 2010; published online II May 2010 the study design and methods are described elsewhere (Million Women Study Collaborative Group, 1999) and the questionnaire can be viewed at http://www.millionwomenstudy.org.

All study participants have been flagged on the National Health Service (NHS) Central Registers and tumour registrations and deaths are routinely notified to the study investigators. This information includes the date of each such event and codes the tumour site and morphology using the 10th revision of the International Classification of Diseases (ICD-10) (World Health Organization, 1992). Follow-up is complete for over $99 \%$ of the study population.

Acoustic neuromas were defined as those coded as ICD-10 D33.3, with morphology code ICD-O 9650/0. Pituitary tumours were defined as those coded as ICD-10 C75.1, D35.2 or D44.3.

Women were classed as current, past or never smokers, as reported at recruitment. Current smokers were further classified according to the average number $(<15,15+)$ of cigarettes smoked per day.

\section{Statistical analysis}

Women were excluded from the analyses if at recruitment they had been diagnosed with any type of invasive tumour (other than nonmelanoma skin cancer [C44]) or any non-invasive tumour of the $\mathrm{CNS}$, or if there was no information on smoking status. Women were also excluded if they reported having the inherited disorder neurofibromatosis (Q85.0) at recruitment.

Eligible women contributed person years from the date of recruitment until the date of registration of an acoustic neuroma or a pituitary tumour, date of death or end of follow-up, whichever was the earliest. In addition, women diagnosed with any cancer 
(except non-melanoma skin cancer) or any non-invasive CNS tumour during the follow-up period were censored at the date of diagnosis of that tumour, to avoid potential biases because of the effect of treatment or of altered surveillance. The end of follow-up for tumour incidence was 31 December 2007 for East Anglia, South West and North West (Mersey) regions, 30 June 2007 for Oxford, Thames, West Midlands and Trent and 31 December 2006 for North Yorkshire, North West (Manchester/Lancashire) and Scotland.

Relative risks (RRs) and 95\% confidence intervals (CIs) were obtained using Cox proportional hazards models with attained age as the underlying time variable. The proportional hazards assumption was assessed using tests based on Schoenfeld residuals, which showed no evidence of a violation for any of the exposure comparisons listed in the tables.

Analyses were stratified by region and by age at recruitment, and as there are no established common environmental risk factors for either tumour site, we examined the effect of adjusting for the following potential risk factors separately, and for all simultaneously: socio-economic status (quintiles based on Townsend deprivation index; Townsend et al, 1988), height $(<160,160-164.9, \geqslant 165 \mathrm{~cm})$, body mass index $(<25,25-29.9$, $\geqslant 30 \mathrm{~kg} \mathrm{~m}^{-2}$ ), alcohol intake (never, $<7, \geqslant 7$ drinks per week), strenuous exercise $(<1,1, \geqslant 2$ times per week), parity (nulliparous, $1-2$ and $3+$ full-term pregnancies), age at first birth $(<20$, $20-24,25+$ years), menopausal status (pre/perimenopausal, $<5$ years postmenopause, $5+$ years postmenopause) and use of oral contraceptives (never, $<5, \geqslant 5$ years of use) and of hormone therapy for the menopause (never, past, current). Women with missing values for any of the adjustment variables were assigned to a separate category for that variable.

\section{RESULTS}

In total, 1240593 women aged 56 years on average at recruitment were included in the analyses. During 10.2 million person years of follow-up (an average of 8.2 years per woman), 177 acoustic neuromas and 174 pituitary tumours were registered. Table 1 shows characteristics of the study population by smoking status. A total of 607215 (49\%) women had ever smoked, and 254992 (21\%) were current smokers.

Women who had ever smoked had a significantly decreased risk of acoustic neuroma compared with never smokers (adjusted $\mathrm{RR}=0.69,95 \% \mathrm{CI}=0.51-0.95, P=0.02$ ). As shown in Table 2, risk was significantly lower in current smokers (RR compared with never smokers $=0.41,95 \% \mathrm{CI}=0.24-0.70, P=0.001)$ than in past smokers (RR compared with never smokers $=0.87,95 \%$ $\mathrm{CI}=0.62-1.22, P=0.4): P$ for heterogeneity $=0.006$. Although the reduction in risk in current smokers seemed to be greater with increasing numbers of cigarettes smoked ( $R R s=0.53$ vs 0.27 in women who smoked $<15$ and $15+$ cigarettes per day, respectively), this difference was not statistically significant ( $P$ for heterogeneity $=0.2)$. Smoking was not associated with incidence of pituitary tumours (RR for ever $v$ s never smokers $=0.99,95 \%$ $\mathrm{CI}=0.73-1.35, P=0.95$; for current $v s$ never smokers, $0.91,95 \%$ $\mathrm{CI}=0.60-1.40, P=0.7)$. No material effect on the RRs was seen

Table I Characteristics of the study population, by smoking status at recruitment

\begin{tabular}{|c|c|c|c|}
\hline \multicolumn{4}{|l|}{ Characteristics at recruitment } \\
\hline Socio-economic group (\% in upper third) & 23 & 33 & 38 \\
\hline Past use of oral contraceptives (\%) & 65 & 64 & 56 \\
\hline Mean parity (s.d.) & 2.3 & 2.1 & 2.1 \\
\hline Mean height, cm (s.d.) & $161.5(6.8)$ & $162.3(6.7)$ & $162.0(6.7)$ \\
\hline Mean alcohol intake, g per week (s.d.) & $47.1(59.6)$ & $53.8(58.7)$ & $37.1(45.8)$ \\
\hline Current use of hormone replacement therapy (\%) & 36 & 36 & 31 \\
\hline \multicolumn{4}{|l|}{ Follow-up for incident acoustic neuromas and pituitary tumours } \\
\hline Person-years of follow-up (millions) & 2.05 & 2.88 & 5.23 \\
\hline Number of incident acoustic neuroma cases & 16 & 52 & 109 \\
\hline Number of incident pituitary tumour cases & 31 & 52 & 91 \\
\hline
\end{tabular}

Table 2 Relative risks (RRs) and 95\% confidence intervals (Cls) for incident acoustic neuroma and pituitary tumour in relation to smoking status in the Million Women Study cohort

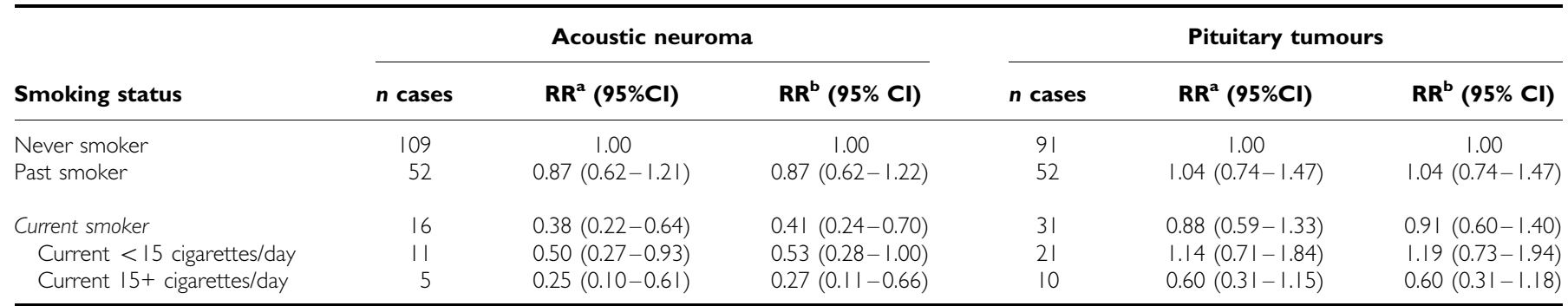

${ }^{\mathrm{a} R R s}$ stratified by region and by age at recruitment, with attained age as the underlying time variable. ${ }^{\mathrm{b}} \mathrm{RRs}$ stratified by region and by age at recruitment, and adjusted for socioeconomic status, alcohol intake, height, body mass index, strenuous exercise, parity, age at first birth, menopausal status and use of oral contraceptives or hormone therapy for the menopause, with attained age as the underlying time variable. 
with either individual or simultaneous (Table 2) adjustment for the 10 potential confounding factors considered.

\section{DISCUSSION}

In this large prospective study, incidence of acoustic neuroma was significantly and substantially decreased in current smokers. Incidence of pituitary tumours was not significantly associated with smoking. Our findings are consistent with the results from the only relevant earlier epidemiological study, a case-control study including 563 acoustic neuromas ( $R R$ for current vs never smokers $=0.5$; Schoemaker et al, 2007) and 299 pituitary tumours (RR for ever $v s$ never smokers $=1.2$; Schoemaker and Swerdlow, 2009).

Strengths of this investigation include the prospective study design, large study size and complete and non-differential followup for cancer incidence. Despite the large sample size, the numbers of cases were still relatively small and the study had limited power to investigate smoking by amount or duration.

\section{REFERENCES}

Asa SL, Ezzat S (2009) The pathogenesis of pituitary tumors. Ann Rev Pathol 4: $97-126$

Benson VS, Pirie K, Green J, Bull D, Casabonne D, Reeves GK, Beral V, for the Million Women Study Collaborators (2010) Hormone replacement therapy and incidence of central nervous system tumours in the Million Women Study. Int J Cancer; [Epub ahead of print]. doi:10.1002/ijc.25184

Benson VS, Pirie K, Green J, Casabonne D, Beral V (2008) Lifestyle factors and primary glioma and meningioma tumours in the Million Women Study cohort. Br J Cancer 99: 185-190

Evans DG, Moran A, King A, Saeed S, Gurusinghe N, Ramsden R (2005) Incidence of vestibular schwannoma and neurofibromatosis 2 in the North West of England over a 10-year period: higher incidence than previously thought. Otol Neurotol 26: $93-97$

Kapoor D, Jones TH (2005) Smoking and hormones in health and endocrine disorders. Eur J Endocrinol 152(4): 491 -499

Mandelzweig L, Novikov I, Sadetzki S (2009) Smoking and risk of glioma: a meta-analysis. Cancer Causes Control 20: 1927-1938
No clear association has been found between smoking and incidence of other tumours of the CNS, including glioma or meningioma, either in this cohort (Benson et al, 2008) or in other studies (Mandelzweig et al, 2009). Possible mechanisms for an association between smoking and risk of acoustic neuroma include, as well as direct effects of tobacco carcinogens, the effects of cigarette smoking on hormonal status (Kapoor and Jones, 2005); female sex hormones may have a function in development of some central nervous system tumours, including acoustic neuroma (Benson et al, 2010). A reduced risk of acoustic neuromas among smokers, if confirmed, would have minimal implications for public health: acoustic neuromas are much rarer than the cancers that are increased among smokers.

\section{ACKNOWLEDGEMENTS}

We thank all of the women who participated in the Million Women Study. This study was supported by Cancer Research UK, the NHS Breast Screening Programme and the Medical Research Council.

\section{Appendix}

Million Women Study Steering Committee

Joan Austoker*, Emily Banks, Valerie Beral, Judith Church, Ruth English, Jane Green, Julietta Patnick, Richard Peto, Gillian Reeves, Martin Vessey and Matthew Wallis.

${ }^{\star}$ Deceased.

\section{Million Women Study Coordinating Centre Staff}

Simon Abbott, Miranda Armstrong, Krys Baker, Angela Balkwill, Vicky Benson, Valerie Beral, Judith Black, Anna Brown, Diana Bull, Benjamin Cairns, James Chivenga, Barbara Crossley, Dave Ewart, Sarah Ewart, Lee Fletcher, Laura Gerrard, Adrian Goodill, Isobel Green, Jane Green, Elizabeth Hilton, Joy Hooley, Sau Wan Kan, Carol Keene, Oksana Kirichek, Nicky Langston, Bette Liu, MariaJose Luque, Maria MacGregor, Lynn Pank, Kirstin Pirie, Gillian Reeves, Emma Sherman, Evie Sherry-Starmer, Moya Simmonds, Elizabeth Spencer, Helena Strange, Siân Sweetland, Alison Timadjer, Sarah Tipper, Ruth Travis, Xiaosi Wang, Joanna Watson, Stephen Williams, Lucy Wright, Tienyu Yang, Heather Young.
McGregor AM (2009) Diagnosis of pituitary disease. Medicine 37: 393-398 Million Women Study Collaborative Group (1999) The Million Women Study: design and characteristics of the study population. Breast Cancer Res 1: 73-80

Propp JM, McCarthy BJ, Davis FG, Preston-Martin S (2006) Descriptive epidemiology of vestibular schwannomas. Neuro Oncol 8: 1-11

Schoemaker M, Swerdlow A (2009) Risk factors for pituitary tumours - a case-control study. Cancer Epidemiol Biomarkers Prev 18: $1492-1500$

Schoemaker MJ, Swerdlow AJ, Auvinen A, Christensen HC, Feychting M, Johansen C, Klaeboe L, Lonn S, Salminen T, Tynes T (2007) Medical history, cigarette smoking and risk of acoustic neuroma: an international case-control study. Int J Cancer 120: 103-110

Townsend P, Phillimore P, Beattie A (1988) Health and Deprivation: Inequality and the North. Croom Helm: London

World Health Organization (1992) International Statistical Classification of Diseases and Related Health Problems, 10th Revision. World Health Organization: Geneva

\section{Collaborating UK NHS Breast Screening Centres}

Avon, Aylesbury, Barnsley, Basingstoke, Bedfordshire and Hertfordshire, Cambridge and Huntingdon, Chelmsford and Colchester, Chester, Cornwall, Crewe, Cumbria, Doncaster, Dorset, East Berkshire, East Cheshire, East Devon, East of Scotland, East Suffolk, East Sussex, Gateshead, Gloucestershire, Great Yarmouth, Hereford and Worcester, Kent, Kings Lynn, Leicestershire, Liverpool, Manchester, Milton Keynes, Newcastle, North Birmingham, North East Scotland, North Lancashire, North Middlesex, North Nottingham, North of Scotland, North Tees, North Yorkshire, Nottingham, Oxford, Portsmouth, Rotherham, Sheffield, Shropshire, Somerset, South Birmingham, South East Scotland, South East Staffordshire, South Derbyshire, South Essex, South Lancashire, South West Scotland, Surrey, Warrington Halton St Helens and Knowsley, Warwickshire Solihull and Coventry, West Berkshire, West Devon, West London, West Suffolk, West Sussex, Wiltshire, Winchester, Wirral, Wycombe. 